\title{
Group Efficacy in Asynchronous vs. Multi-synchronous Virtual Teams: An Empirical Study
}

\author{
Yingxin Pan and Chen Zhao \\ IBM China Research Lab, Bldg. 19, Zhongguancun Software Park, \\ Beijing, P.R. China, 100094 \\ \{panyingx, zhaochen\}@en.ibm.com
}

\begin{abstract}
Group efficacy has begun to receive more attention in HCI. The paper describes a mixed-design experiment aimed to explore the effect of time on group efficacy development in two computer-mediated virtual conditions: asynchronous vs. multi-synchronous teams. The relationships of group efficacy measures at different times and tool evaluation, team satisfaction and performance are also explored. Forty university students who participate in the study are administered questionnaires over the course of the assigned ten-day task. Results show group efficacy changes as a function of time in the asynchronous environment. In addition, the positive relations between group efficacy and tool evaluation and adoption and team satisfaction were demonstrated for asynchronous groups. The findings indicate group efficacy is dynamic with team development and useful in signaling how technology and collaboration modes impact user experience and interaction in the asynchronous environment.
\end{abstract}

Keywords: Group Efficacy, Asynchronous \& Multi-synchronous communication, groupware, virtual team, CSCW.

\section{Introduction}

Efficacy beliefs are constructed as future-oriented judgments about capabilities to pursue a course of action to meet given situational demands. Since Bandura introduced the concept of self-efficacy perceptions, research in many arenas has demonstrated the power of efficacy judgments in human learning, performance and motivation [7]. An extension of Bandura's self-efficacy is collective or group efficacy which captures a member's beliefs about the capacity of a group or organization. Although both self- and group efficacy are widely recognized in many fields, in human-computer interaction community, efficacy theories have been largely unnoticed [11]. Ramalingam et al. reported that self-efficacy in computing could be used as a predictor of technology learning and achievement [17]. Recent work has begun to make explorations into group efficacy. Carroll et al.'s work provided evidence that community collective efficacy is a valid construct in community computing domain. Still, the collective efficacy of workgroups in computer-mediated interaction has not been well studied yet. Of particular interest is the development and 
role of group efficacy over time in today's virtual groups which are more relying on technologies, as the beliefs of group members evolve with the group development [1] and have been proved effective in predicting future group achievement in traditional teams.

During the course of a project or a task, group members adjust their judgments on how well their group can achieve, while in previous studies group efficacy is often measured at a separate point of time during a task and then used as a general indicator of the collective expectation of future performance. Baker's study found that group efficacy changes over time as group receives feedback in traditional small task groups [1]. However, for virtual teams the topic of group efficacy development is left open. To better understand group efficacy in virtual teams, two typical virtual collaborative modes are identified in the study: asynchronous vs. multi-synchronous. Asynchronous communication has many advantages to enable dispersed collaboration, whereas prolonged response and weak awareness of people and events make the newly-formed groups hard to build swift trust which is closely related to group efficacy [5]. Multisynchronous collaboration seemed to have benefits to overcome the difficulties, but it is often made up of scattered working environments or tools. This sometimes may lead to such a problem as information chaos [16]. Therefore, it is worth the research effort on how group efficacy differs and develops in the two virtual environments supported by technologies.

Another relatively untouched topic is the effect of group efficacy for understanding human-computer interaction. It is known that an individual's interaction with or through a computer is directly influenced by how well the technology facilitates their abilities [11]. In virtual group contexts, people interact with each other by means of different sophisticated technologies. Group efficacy is expected to be a valuable measure for assessing the consequences that computer-mediated tools have on the starting capabilities of a group [4].

In this paper, therefore, group efficacy was investigated in two comparative conditions - asynchronous and multi-synchronous environments of virtual teams. The study explored two questions: 1) does group efficacy perception change as a virtual team develops and how; and 2) how well group efficacy measures at different times indicate technology impact on virtual team results and interaction?

\section{Group Efficacy}

\subsection{Group Efficacy Development and Measurement}

Group efficacy has been defined as the group members' collective estimate of the group's ability to perform a specific task. Like self-efficacy, group efficacy differs from general confidence because confidence is a general affective state, whereas efficacy is extremely task specific [15]. When a task starts that requires much team interdependence, team members have the opportunity to develop shared mental models and to use this shared knowledge to guide their behavior.

So when team members set out to do a specific task, how is group efficacy initially developed? Bandura [2] proposed four sources of information that lead to the development of self efficacy. They are past performance accomplishment, vicarious 
experience, verbal persuasion and physiological and emotional arousal respectively. Other than these aforementioned sources of information on self-efficacy, its effect on team results can also be moderated by other variables, for example, complexity of the task and clarity of goals [18]. Along the same train of thoughts, researchers began to explore antecedents of group efficacy, although Bandura addressed this issue by stating that self- and collective efficacy shared the similar sources [2]. For example, Pescosolido [15] found a strong link between the informal leaders' perceptions of efficacy and group-level measurements of efficacy. In Watson et al.'s study [21], collective efficacy was found to be influenced by both individual-level variables such as self-efficacy, optimism and group-level variables of group size, past team performance and confident leadership.

However, actually many real tasks or projects do take some time to complete and teams go through development cycles. Apart from external variables, time itself has an effect on how group efficacy develops as team develops. Some studies have explored whether certain variables have an effect on collective beliefs over time. Middup et al.'s [11] empirical work showed how group exercises and group memories of activities affect self- and group efficacy as a function of time. As Baker [1] claimed, the beliefs of group members have a dynamic quality and evolve with the development of the group. Especially when a group is confronted with a new situation to perform a task, group efficacy could reasonably be assumed dynamic as time goes and at the same time based on how members react to the situation. Therefore, how group efficacy develops in virtual teams with different collaboration modes deserves research.

With respect to efficacy measurement, there has been generated much literature in clarifying and testing different methods of measurements. Generally, there exist three major types of methods. The first method is group-level aggregation of individual self-efficacy beliefs. The second is group-level aggregation of individual group efficacy beliefs and the third way is conducted by achieving group consensus. All these methods have their own pros and cons. Researchers have attempted to see which one is more predictive in specific contexts. Whiteoak et al. [22] compared the three methods of assessing group efficacy in a laboratory study. The conclusion is the three methods didn't differ - neither in terms of their capacity of discriminating high and moderate task-difficulty conditions, nor in their consistency, magnitude of their relationship with goals and the extent to which they are influenced by performance. In Hardin et al.'s study [8] of measuring group efficacy in virtual teams, these methods differed: the aggregated method had greater predictability than a group consensus method.

Nevertheless, no matter which method is chosen, there is another issue that needs to be taken into consideration. As stated in the aforementioned part, group efficacy as a collective belief is of the dynamic quality. When a team is first formed, team members can hardly form a common understanding or belief simultaneously. They know little about what each other are like, how competent they are, etc. Their roles in the team and the procedures for interaction may be unclear as well. Hence the expectations of members of newly formed teams are somewhat blurry or even inaccurate. Their perceptions of group efficacy for performance may be primarily dependent on perceptions of individual self-efficacy. Baker [1] held the same argument on this. If members don't share expectations about team outcomes, group 
efficacy is not likely to exist. So the measurement of group efficacy at the very beginning of team forming or a task might be not that appropriate or valid in predicting team outcomes, particularly when there is much uncertainty either in terms of the task or the interaction environment. Therefore, at what time the assessment of group efficacy would be most effective is worth exploring. In virtual teams who are rarely meet face-to-face, various technologies or tools are employed to facilitate communication. Shared understanding of the group's capabilities couldn't be generated at the very outset. Technologies may enhance or impede the communication or even team building. As a result, the question is to be answered: when group efficacy is formed and effectively predicts group outcomes in computermediated collaborative virtual teams?

Based on this logic, this paper tried to explore how group efficacy develops during the cycle of team development and see how it may change as a function of time in two different collaborative HCI virtual environments.

\subsection{Group Efficacy with Group Outcomes}

Numerous studies have proved the strong linkage between performance and selfefficacy. Researchers also found the positive relationship of group efficacy with a variety of group and organizational outcomes. With a plenty of research done in traditional teams and its consistent ability to predict performance, many researchers began to make extension into virtual team environment [20] and community computing domain [11]. How technology enhances or hinders people in working or collaborating through human interaction with computer is one of the fundamental issues in HCI. In Carroll et al.'s work [4], community collective efficacy is proposed to be useful for understanding the experiences of people using a community network. Hardin et al.'s study [8] called for more efforts to investigate the relationship between group efficacy and virtual team performance which is actually lacking in current research. Nevertheless, how group efficacy can work to indicate group outcomes, particularly technology impact, is still deficient.

In this paper, the relationship of group efficacy with such group outcome variables as group satisfaction, technology evaluation and adoption were investigated.

\section{Asynchronous vs. Multi-synchronous Virtual Teams}

Virtual or distributed teams are formed for any number of reasons, from educational purposes, to business process redesign or resources reallocation. They perform projects or tasks with a host of constantly emerging technologies. Broadly speaking, there are two fundamentally different approaches to network-based collaboration for virtual teams [19]. Team workspace or asynchronous applications provide an electronic space where members of distributed task groups can share and edit documents and other data, chart the progress of tasks, and communicate in threaded discussions. Synchronous is all about communicating in the present. Examples of this synchronous communication include a telephone conversation, video conferencing and instant messaging. However, practical work often involves more complex situations, as work is a cycle of divergence and convergence [12]. Multi-synchronous environment is 
consequently becoming desirable where direct communication and asynchronous means are combined to satisfy varying needs of people.

There are two major important distinctions between virtual teams and traditional teams [8]. One is lack of collocation and the other is the need to use sophisticated information technology [10]. These two factors add complexity to team interactions. It is not surprising how a rich array of cues normally experienced in co-located teams is absent during distributed work. In a face-to-face environment, the cues such as body language and vocal inflection are easily accessed that even the most highfidelity video conferencing systems cannot fully reproduce. In a virtual environment, participants have no such access, where diminished trust could be caused. Closely related to trust is group efficacy within teams [3]. Some researchers have suggested that important aspects of group efficacy include shared beliefs in the team's abilities at communication and perceived competence for coordinated group activities [14]. Distributed interaction may hinder the development of this efficacy within teams [3]. This issue seemed more obvious for asynchronous communication in that normal interaction with human cues gets more difficult. Multi-synchronous collaboration makes up for what asynchronous may miss, but scattered working environments or tools can engender other problems, such as information chaos [16] which can increase task load. In terms of the impact of information technology in virtual teams, group efficacy construct as a simple method is promising to be useful to investigate the issue of how technology really works in HCI.

Group efficacy in computer-mediated virtual groups has begun to receive more attention [13, 17] but is not well studied yet. It is worthwhile to make further exploration to see how group efficacy develops over time and how it indicate technology impact and group outcomes in different collaborative modes of virtual working.

\section{Method}

\subsection{Participants and Design}

In total, forty Chinese university students participated in the study as members of 5 experimental and 5 control workgroups performing the same assigned task. Each group was made up of 4 students who are all senior undergraduates and master students. The 5 experimental groups consisted of 11 males and 9 females and control groups of 12 males and 8 females. All groups were of mixed sex. The participation was voluntary. The students didn't know each other before. They were encouraged to make efforts and would be given gifts of different prices - better final performance will be acknowledged by higher-value gifts as recognition to their team.

In experimental groups, participants were required only to use a web-based asynchronous communication platform - Lotus Notes TeamRoom. Besides TeamRoom, The 5 control groups could use other real-time and asynchronous communication tools including ICQ, MSN, emails and phone calls, except face-toface, which created a multi-synchronous environment. This design ensures the two types of groups both simulate virtual working environments. 
The task is to explore eastern-western cultural differences and thus to provide suggestions to people in dealing with cultural affairs. The task was chosen for a few reasons: firstly, it is a task involving both brainstorming and problem-solving; secondly, there is no universal description of eastern-western cultural differences and how they could be categorized. Participants can contribute whatever they think makes sense from any perspective, no matter what academic background they are of. Third, the task also involves document sharing and management which made it closer to real working scenarios. In the task, participants were asked to: (1) discover possible cultural differences and categorize them in a way they prove reasonable, (2) compose a report based on the work (Deliverable 1) and (3) make course slides to present the findings and provide implications and tips in cross-cultural affairs (Deliverable 2).

The task duration is ten-day for each group. It's not very long, nor short. But there is no literature that specifies the minimum or maximum duration of a short duration team. Of the 50 teams studied by Tuckman [20] investigating team development, teaming durations ranged from 1 hour to a few years. Considering the task load and complexity and according to team results, ten days were appropriate.

\subsection{Procedure and Technology}

Participants of each group met face to face on the first day for about 1.5 hours to get to know their team members. Also, the kick-off meeting served other purposes of: (1) signing agreements by participants, promising they would commit themselves to their team performance; (2) providing training on basic functions of Notes TeamRoom for about 30 minutes; each group had one private TeamRoom which only the four team members and the experimenter could access. They were randomly assigned a user id and password for logging on; (3) providing participants with the instructions about the task, procedures and experiment requirements; (4) filling in the group efficacy questionnaire for the first time at the end of the meeting and (5) sending the first thank-you gifts to all of them for their interest in joining the study. Participants were strongly encouraged by the instructor to work on the task collaboratively.

During the ten days, participants were requested to deliver two results as mentioned above which the $2^{\text {nd }}$ deliverable would have a heavier weight for their final performance. Participants were encouraged to reach their agreement on the categories cultural differences fell into before they worked out the $1^{\text {st }}$ report. Participants only needed to submit deliverable 1 (by the $6^{\text {th }}$ day) and 2 (by the $10^{\text {th }}$ day). By manipulating the task, participants had a better sense of time and deadline so that they wouldn't rush just in the last few days to meet the deadline. The work was delivered to the experimenter via any electronic means.

To approximately match the four team development phases, group efficacy was assessed for four times over the ten-day span. Participants were required to finish the group efficacy questionnaire for another three times in addition to the first measurement at the kick-off meeting: at day 4, day 7 and day 10. The exact time on these days to complete it was not the same, slightly moderated according to their progress. All questionnaires were administered and collected by email except the first completion. Participants would finish two more questionnaires about team satisfaction and TeamRoom evaluation when the task is completed. In addition, TeamRoom data, chatting logs, exchanged emails and other task-relevant communication information, together with their individual self-reports, should be kept and submitted. 
TeamRoom 7.0 used in the study is a Lotus Domino application, accessible via a web browser. It allows for the creation of threaded, yet asynchronous, discussion. Information and documents could also be easily shared. Each group owned a new and independent TeamRoom which was inaccessible to other groups.

\subsection{Measures}

Group Efficacy: In this study, group efficacy was measured by individual-level assessment of group efficacy beliefs [23]. As group efficacy is specific to a domain or a group task, a task-specific eleven-item scale was developed. The sample items are: "I believe that our group has the ability to find the key cultural differences" and "I believe that our group has the ability to conduct effective discussion to reach good results". All items were completed on an 11-point scale $(0=$ not at all confident, $5=$ moderately confident to $10=$ totally confident). Nevertheless, to determine if it is appropriate to aggregate the individual beliefs when investigating the relationships between group efficacy and group-level outcomes, the within-group interrater reliability should be calculated to assess the level of agreement within groups on the group efficacy measure [22]. In this study, the Kendall's concordance coefficients were for group efficacy .91, .92, .96 and .96 at time1, time2, time 3 and time4 respectively. The values demonstrated that the group-level analysis of efficacy was meaningful.

Group Satisfaction: Three items were developed to measure group satisfaction on 5point scale after task is finished. The sample item is: "In all, how satisfied are you with your group?" ( $1=$ completely dissatisfied to $5=$ completely satisfied $)$. Cronbach's alpha is .8085. ${ }^{1}$

TeamRoom Evaluation: Three items were used to assess the users' ratings of TeamRoom on 5-point scale after task is finished. The sample items are: "Based on this experience, how do you like TeamRoom?" (1= completely dislike to $5=$ completely like) and "How much possibility will you choose to use TeamRoom in other situations?" ( $1=$ never to $5=$ always $)$. Cronbach's alpha is .8028 .

Group Performance: Two independent evaluators who were expert at cross-cultural issues assessed the final report and course slide from each group. Both the report and the slides were rated (full score is 10) and their weights were .3 and .7 respectively. The final performance was calculated by averaging the two scores given by two evaluators.

\section{Results}

All groups completed the task on time and each delivered one report and course presentation slides. TeamRoom data showed members of experimental groups had asynchronous group discussion, sharing documents, made team announcements, wrote the team report and edited TeamRoom settings. Of the five control groups,

\footnotetext{
${ }^{1}$ As a measure of the reliability of psychometric instrument, Cronbach's alpha indicates the extent to which a set of test items can be treated as measuring a single latent variable.
} 
three groups chose to use TeamRoom mostly to share documents. Of the two groups who didn't use TeamRoom, one registered a public mailbox where they could share documents and post chat logs and the other just used individual emails to share documents. Other than TeamRoom, self-reports and experiment-related data submitted by control groups revealed that they communicated by means of ICQ, MSN, email and text messaging over cell phone. They relied more on real-time communication tools, mostly ICQ which is the most popular synchronous communication software in China and occasionally MSN to perform the task. Four control groups built a team-shared ICQ discussion room. Members would discuss at the same time in the room. One control group just communicate one-to-one by ICQ and sent out the discussion results and pushed the progress via emails. A few participants from control groups used text messaging of cell phones to make notifications for discussion. Although control groups were given much freedom, they still chose to use what they are used to: they use emails and real-time communication tools all the time. Overall, the two conditions were both as designed: asynchronous vs. multi-synchronous virtual teams.

To test the effects of time on group efficacy development in experimental and control groups, a repeated measurement MANOVA was carried out with one between-group variable - condition (asynchronous vs. multi-synchronous condition) and one within-group variable (time). Group efficacy belief was used as dependent variable. Results showed that the interaction effect of time * condition was significant $(\mathrm{F}=6.062, p<.05$, see figure 1). Subsequent univariate tests showed that the differences of group efficacy between time1 and time2, time 2 and time 4 were significant $(p<.05)$ and the difference between time 1 and time $3(p=.055)$ was marginally significant in experimental groups. Group efficacy didn't differ significantly over time in control groups (see table 1 ).

Results also showed that both time $(\mathrm{F}=3.373, p<.05)$ and condition $(\mathrm{F}=6.062$, $p<.05)$ had a significant main effect on group efficacy. Subsequent univariate tests revealed that at time 2 and time 3, experimental and control group in the two comparative conditions revealed significant differences in their collective efficacy beliefs $(p<.05)$. Control groups showed a significantly higher level of group efficacy than experimental group. That is to say, when the team members started working in two different conditions, group efficacy as a collective estimate in group's ability to perform the task differed. At time 1, the two conditions showed no significant difference in group efficacy beliefs. This indicated that the sampling was valid and the two groups had no pre-study bias in group efficacy. At time 4 when the task was approaching the end, their group efficacy estimations didn't differ significantly either.

Prior to relating group efficacy with group-level outcomes, a $t$-test was conducted to see if these variables in two conditions differed: TeamRoom evaluation, team satisfaction, performance and TeamRoom Adoption for which the following two indicators were used. Function Usage is calculated by the number of TeamRoom functions used; Content Contribution is assessed by the number of postings posted in TeamRoom. The results were presented in table $2(\mathrm{~N}=5$ for experimental groups; $\mathrm{N}=$ 5 for control groups for team satisfaction and performance and $\mathrm{N}=3$ for $\mathrm{TR}$ Evaluation \& using patterns). Except for the TeamRoom Adoption, all the other three variables didn't show significant differences. As expected, experimental groups made a much more frequent use of TeamRoom in terms of content contribution and function usage. 


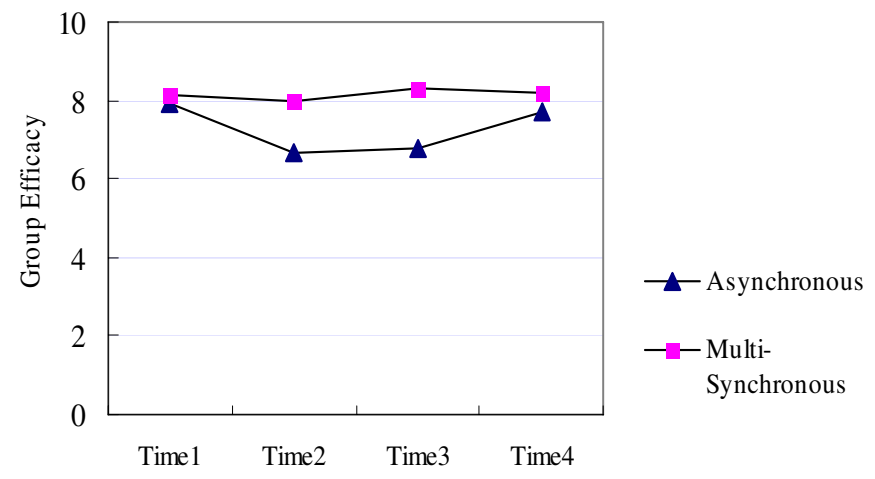

Fig. 1. Group Efficacy Development with Time in two Conditions $(\mathrm{N}=40)$

Table 1. Between-group comparison of Group Efficacy scores ( $\mathrm{N}=20$ for each condition)

\begin{tabular}{ccccc}
\hline & \multicolumn{2}{c}{ Experiment Group } & \multicolumn{2}{c}{ Control Group } \\
\cline { 2 - 5 } & \multicolumn{2}{c}{$\mathrm{M}$} & $\mathrm{SD}$ & \multicolumn{2}{c}{$\mathrm{M}$} & $\mathrm{SD}$ \\
\hline Time 1 & 7.95 & 0.75 & 8.13 & 1.31 \\
Time 2 & 6.69 & 1.09 & 7.98 & 1.08 \\
Time 3 & 6.75 & 1.26 & 8.32 & 0.51 \\
Time 4 & 7.70 & 1.21 & 8.18 & 0.97 \\
\hline
\end{tabular}

Finally, to test how group efficacy correlates with team outcomes and to explore how the measurements of group efficacy at different times would better correlate with team outcomes, two correlation analyses were made respectively for the two conditions. All the correlations were based on aggregated data at the group level. Table 3 presented the results of experimental groups.

Table 2. Between-group tests of group outcomes

\begin{tabular}{ccccc}
\hline & \multicolumn{2}{c}{ Experimental Group } & \multicolumn{2}{c}{ Control Group } \\
\cline { 2 - 5 } & $\mathrm{M}$ & $\mathrm{SD}$ & $\mathrm{M}$ & $\mathrm{SD}$ \\
\hline TR Evaluation & 10.15 & 1.58 & 9.55 & 1.31 \\
Team Satisfaction & 11.80 & 1.41 & 11.90 & 1.17 \\
Content Contribution* & 80.80 & 34.82 & 19.20 & 0.51 \\
Function Usage* & 7.80 & 2.17 & 2.40 & 1.51 \\
Team Performance & 7.67 & 0.96 & 7.73 & 0.85 \\
$* p<.01$ & & & &
\end{tabular}

First the table for experimental groups was interpreted row by row (table 3). Overall, group efficacy measurement was quite effective in indicating how participants think of the tool they used in performing the task, as the correlations with TeamRoom evaluation were all significant at time 2, 3 and 4 . Team satisfaction was significantly correlated with the measurements of group efficacy at time 2 and 4 . 
Group efficacy levels at time 2 and time 4 were also found to be significantly correlated with content contribution; and for function usage, the relationship with the group efficacy at time 4 was significant. All the significant relationships were positive. The higher group efficacy estimation, the more usage of TeamRoom and the higher team satisfaction level. Performance was significantly correlated with no one of the four group efficacy measurements. For this small sample size, many other factors may have taken effect.

Then the results were interpreted by column (table 3). The correlation between the group efficacy measurement at time 1 and all team variables didn't achieve a significant level. It means the initial measurement of collective beliefs at the outset of a task was not valid or accurate to predict outcome variables. Basically, the measurement at time 4 has the best power to predict group-level variables. But since time 4 is often too close to the end of a task, the practical value may be weakened. Group efficacy measurement at time 2 turned out to be an alternative as it was significantly correlated with 3 of the group variables including team satisfaction and TeamRoom evaluation. For the measurement at time 3, the result was not very strong. The GE_Avg. was a simple average calculation of the four-time measurements. Its power was not distinctively stronger than that of other separate assessments. Thus it can be concluded that group efficacy was effective in reflecting some group-level outcomes in the asynchronous condition.

However, the correlation results in control groups were totally different. The relationships of all pairs of the variables were not significant. It showed group efficacy measurement were invalid in indicating group outcomes in the multisynchronous environment.

Table 3. Correlations between group efficacy and team outcomes for experimental groups $(\mathrm{N}=5)$

\begin{tabular}{cccccc}
\hline & GE_t1 & GE_t2 & GE_t3 & GE_t4 & GE_Avg. \\
\hline TR Evaluation & .374 & $\mathbf{. 9 7 6 *}$ &. $\mathbf{8 7 6} * * *$ & $\mathbf{. 9 1 1 * *}$ & $\mathbf{. 9 2 2 *}$ \\
Team Satisfaction & -.082 & $\mathbf{. 8 2 3} * *$ & .584 & $\mathbf{. 8 3 9} * *$ & .703 \\
Content Contribution & .444 & $\mathbf{. 8 3 8} * * *$ & .633 & $\mathbf{. 8 5 6} * * *$ & $\mathbf{. 9 0 6 * *}$ \\
Function Adoption & .455 & .785 & .586 & $\mathbf{. 8 4 0} * * *$ & $\mathbf{. 8 9 4} * *$ \\
Team Performance & -.742 & -.383 & -.657 & -.208 & -.504 \\
\hline
\end{tabular}

Note: Given a sample size of 5 , it was necessary to set the significance level at .10 and even using the level of .10, power was only sufficient for detecting large effects ( $r>=.8$ ) [8].

$* p<.01 . * * p<.05 . * * * p<.10$.

\section{Discussion}

It has been part of life for people to use different information technologies such as email, groupware and real-time communication software to interact with copartners doing their daily work or perform tasks. Virtual teams have taken advantages of technology advancements and become popular in current organizations. This paper 
has presented an empirical study to explore how group efficacy developed over time and related with group outcomes in two kinds of typical virtual team computermediated environments. Results indicated that group efficacy changed over time in asynchronous condition and that group efficacy assessments differed across two comparative conditions. Group efficacy was found to be significantly correlated with TeamRoom rating and adoption and team satisfaction in asynchronous condition as well.

In the experimental condition where participants were constrained to work completely in a new groupware-supported environment, very interesting results were discovered. Group members experienced an abrupt drop at time 2 shortly after they formed a new team to start the task. According to team development theory, time2 during the task should be closest to the storming status [20]. In the storing phase, members began to realize the task is different or more difficult than they previously imagined. Anxious about progress, members might argue about what actions the group should take. And this situation was exacerbated especially when they were required to communicate and perform by a strange groupware system. It demanded extra efforts for them to get used to the system, and change their familiar ways to interact with other members. Experimental group members have tried to make most use of the shared space: having asynchronous threaded discussions, uploading and downloading documents, publishing team announcements and setting milestones. In addition, lack of immediacy prevented the group from clarifying status quo quickly; lack of personal interactions made it difficult for team building. Trust was weak. As members knew TeamRoom for the first time, it took time for them to know how to use it to push things forward. Collective beliefs in their group capability and future performance at that time turned weak. That's the probable reason why the groupwaresupported groups experienced such a steep drop at time 2, 3 days later from time 1 when they met each other on the first day. The situation continued to time 3 . After that, experimental groups recovered their efficacy beliefs gradually and had almost the same group efficacy estimation with control groups.

As opposed to that, control groups didn't share this pattern, although three out of the five groups used TeamRoom as well. As data indicated, control group members took TeamRoom largely as supplementary. The volume of TeamRoom content contribution and number of functions used by control groups were significantly smaller than those of experimental groups. They used TeamRoom more for document sharing than for discussion and further task management. So the new tool could be reasonably considered to have not much impact on the overall progress. Control group members did the task mostly in a way they usually did: scheduling on-line discussion with familiar real-time communication tools, sharing documents by email and notifying occurrences by cell phone message, etc. Despite no face-to-face meeting, the multiple ways of communication and frequent on-line interaction decreased the spatial and psychological distance among members. Across the ten days, group efficacy didn't show significant change within control groups.

However, the two groups did show significant differences in their group efficacy ratings at time 2 and time3: control groups exhibited a higher level of group efficacy than experimental groups. This result can be explained somewhat from the above 
description. What's more, as Bandura stated, one of the four sources of group efficacy is physiological and emotional arousal [2]. In the asynchronous environment where are solely supported by a new technology, the prolonged response and unfamiliarity with the working environment were likely to strengthen members' perception of task difficulty. This also would lead to negative emotions that decreased efficacy estimation.

Group efficacy was found to be significantly correlated with several group-level outcomes in experimental condition. TeamRoom evaluation ratings and adoption degree (content contribution and function usage) were significantly correlated with group efficacy. The higher level of group efficacy indicated a better evaluation of the tool and a stronger using inclination for the tool. Also, the higher group efficacy estimation means better group satisfaction. It validated the usefulness of group efficacy construct in possible longitudinal studies of technology adoption and impact [4]. From the study, it can be concluded that group efficacy is effective to indicate if users like the technology or if it works well when the technology really comes into play for interaction, at least in an asynchronous condition. Another interesting result is that the first measurement of group efficacy seemed invalid to signal team outcomes. It implies using group efficacy as a predictor, too early measurement would be of little use. Group efficacy measurement at time 2 was proved to be a good indicator as time 4 is often too late to make sense. In contrast again, control groups didn't get any significant correlation.

There is no significant relationship found between group efficacy and performance. Actually the paper is an initial exploration to examine the relationship between group efficacy and virtual team performance according to Hardin et al. [8]. Although strong links between group efficacy and performance among collocated teams has been established, the same relationship has not been established in virtual teams. The two important group outcomes - team satisfaction and performance, didn't demonstrate any significant difference between experimental and control groups either. A few possible explanations could be presented here. First, the small sample made the result not very strong. Second, task performance could be easily affected by other factors. For example, experimental group members had less personal interaction, which might make group communication more task-focused. Or some group might more depend on one or two members' exceptional capabilities instead of collective efforts. Third, as it was actually a "lab" study, participants joined the study voluntarily and had no steadfast obligation to perform the task to the utmost. These factors also pointed out the limitations that should be noted for the paper.

To sum up, group efficacy changes over time in asynchronous virtual teams, and is proved to be effective in investigating how technology impacts human-computer interaction and indicating technology adoption, especially when technology remarkably alters the way people usually work rather than just plays a minor role. In similar working contexts, group efficacy can be used as a signal to show if the environment serves collaboration well by avoiding the drop at the storming phase. Future research is needed to do deeper explorations with field data, e.g., practical virtual project teams, and to analyze the specific technology impacts on collaborative activities. The relationship of group efficacy and performance in virtual teams with the effect of technology should be investigated in more details. 


\section{References}

1. Baker, D.F.: The Development of Collective Efficacy in Small Task Groups. Small Group Research 32(4), 451-474 (2001)

2. Bandura, A.A.: Self-efficacy: The Exercise of Control. Freeman, New York (1997)

3. Berry, G.R.: Can Computer-mediated Asynchronous Communication Improve Team Processes and Decision Making? Journal of Business Communication 43(4), 344-366 (2006)

4. Carroll, J.M., Rosson, M.B., Zhou, J.Y.: Collective Efficacy as a Measure of Community. In: CHI 2005, Portland, Oregon, USA, April 2-7, pp. 1-10 (2005)

5. Fiore, S.M., McDaniel, R.: Building Bridges: Connecting Virtual Teams Using Narrative and Technology. THEN 3 (2006)

6. Gibson, C.B.: Do They Do What They Believe They Can? Group Efficacy and Group Performance across Tasks and Cultures. Academy of Management Journal 42, 138-152 (1999)

7. Goddard, R.D., Hoy, W.K., Hoy, A.W.: Collective Efficacy Beliefs: Theoretical Developments, Empirical Evidence, and Future Directions. Educational Researcher 33(3), 3-13 (2004)

8. Hardin, A.M., Fuller, M.A., Valacich, J.S.: Measuring Group Efficacy in Virtual Teams: New Questions in an Old Debate. Small Group Research 37(1), 65-85 (2006)

9. Hardin, A.M.: Testing the Influence of Collective Efficacy Beliefs on Group Level Performance Metrics: An Investigation of the Virtual Team Efficacy-Performance Relationship in Global Information Systems Project Management Teams. Washington State University MIS Brown Bag Series, Pullman, Washington (2005)

10. Lipnack, J., Stamps, J.: Virtual Teams: People Working Across Boundaries with Technology. John Wiley and Sons, New York (2000)

11. Middup, C.P., Johnson, P.: Towards Using Technological Support of Group Memory in Problem-Solving Situations to Improve Self and Collective Efficacy. In: Proceedings of the 39th Hawaii International Conference on System Science, pp. 1-10 (2006)

12. Molli, P., Skaf-Molli, H., Oster, S., Jourdain, S.: Sams: Synchronous, asynchronous, multisynchronous environments. In: The Seventh International Conference on CSCW in Design, Rio de Janeiro, Brazil (2002)

13. Neale, D.C., Carroll, J.M., Rosson, M.B.: Evaluation Computer-supported Cooperative Work: Models and Frameworks. In: Proceedings of CSCW, Chicago, November 8-10, pp. 368-377. ACM, New York (2004)

14. Paskevich, D.M., Brawley, L.R., Dorsch, K.D., Widmeyer, W.N.: Relationship between Collective Efficacy and Team Cohesion: Conceptual and Measurement Issues. Group Dynamics 3, 210-222 (1999)

15. Pescosolido, A.T.: Informal Leaders and the Development of Group Efficacy. Small Group Research 32(1), 74-93 (2001)

16. Qu, C.T., Nejdl, W.: Constructing a Web-based Asynchronous and Synchronous Collaboration Environment using WebDAV and Lotus Sametime. In: SIGUCCS '01, Portland, Oregon, USA (2001)

17. Ramalingam, V., Wiedenbeck, S.: Development and Validation of Scores on a Computer Programming Self-efficacy Scale and Group Analysis of Novice Programmer Selfefficacy. Journal of Educational Computing Research 19(4), 365-379 (1998)

18. Stajkovic, A.D., Luthans, F.: Self-efficacy and Work-related Performance: A Metaanalysis. Pschological Bulletin 124(2), 240-261 (1998) 
19. Stevenson, T.: Synchronous and Asynchronous Collaboration...Collaborate (2005), VoIP Planet Webcast: www.voipplanet.com

20. Tuckman, B.W.: Developmental Sequence in Small Groups. Psychological Bulletin 63, 384-389 (1965)

21. Watson, C.B., Chemers, M.M., Preiser, N.: Collective Efficacy: A Multilevel Analysis. Personality and Social Psychology Bulletin 27(8), 1057-1068 (2001)

22. Whiteoak, J.W., Chalip, L., Hort, L.K.: Assessing Group Efficacy: Comparing Three Methods of Measurement. Small Group Research 35(2), 158-173 (2004)

23. Zellars, K.L., Hochwarter, W.A., Perrewe, P.L., Miles, A.K., Kiewitz, C.: Beyond selfefficacy: Interactive effects of role conflict and perceived collective efficacy. Journal of Managerial 13, 483-499 (2001) 